\title{
Comparative transcriptome analysis of Syringa vulgaris vegetative apices in vivo and in vitro
}

\author{
A.A. Krinitsina ${ }^{1,4 *}$, A.S. Speranskaya ${ }^{1}$, O.A. Churikova ${ }^{1}$, M.D. Logacheva ${ }^{1}$, E.A. Konorov ${ }^{2,5}$, M.S. Belenikin ${ }^{3}$ \\ ${ }^{1}$ Lomonosov Moscow State University, Moscow, Russia \\ ${ }^{2}$ Vavilov Institute of General Genetics, Moscow, Russia \\ ${ }^{3}$ The Moscow Institute of Physics and Technology, Dolgoprudny, Russia \\ ${ }^{4}$ I.M. Sechenov First Moscow State Medical University, Pharmaceutical Natural Science Department, Moscow, Russia \\ ${ }^{5}$ V.M. Gorbatov Federal Research Center for Food Systems of Russian Academy of Sciences, Moscow, Russia
}

\section{DOI 10.18699/ICG-PlantGen2019-53}

(c) Autors, 2019

*e-mail:ankrina@gmail.com

\begin{abstract}
Long-term conservation of plants in vitro means setting up conditions in which plants slow down their growth, which reduces the frequency of subculture needed to keep the material alive. Fall of cultivation temperature is frequently used for preservation of ornamental plants' cultures and of lilac's cultivars as well. For a better understanding of processes in in vitro culture, a comparative transcriptome analysis of vegetative apices of Syringa vulgaris $L$. during dormancy or in the phase of active growth in vivo and in vitro was carried out. A pairwise comparison of samples showed that a decrease in the temperature of lilac's microshoot cultivation down to $+10^{\circ} \mathrm{C}$ leads to the formation of a response similar to the response to oxidative stress. The changes in gene expression similar to such stress response persist within active shoots growth in sterile culture after their transfer to standard cultivation conditions $\left(+22^{\circ} \mathrm{C}\right)$.

Key words: in vitro conservation; transcriptome; lilac; apex; ornamental plants.
\end{abstract}

\section{Introduction}

Long-term conservation of plants in vitro means setting up conditions in which plants slow down their growth, which reduces the frequency of subculture needed to keep the material alive. Such an effect can be achieved through different approaches. The most common is by decreasing the temperature of cultivation, which leads to a strong slowdown of plant growth (up to its complete stop) and thus makes it possible to reduce the frequency of subculture. This way is widely used for ornamental plants as well (Ozudogru et al., 2010). Different types of explants can be placed into slow growth storage: microshoots or their parts, for example single-noded grafts, buds encapsulated in alginate beads.

Lilac is a widespread ornamental shrub. Nowadays, according to the International Lilac Society, there are more than 2000 cultivars of this genus in the world. The methods of microclonal propagation are being developed and constantly improved for it (Molkanova et al., 2002; Gabryszewska, 2011). It was showed that at a temperature of $+15^{\circ} \mathrm{C}$ the growth of lilac microshoots in vitro is slowing down (Gabryszewska, 2011). While creating the meristem's alginate beads, it is possible to conserve them at a temperature of $+4{ }^{\circ} \mathrm{C}$ (Refouvelet et al., 1998). We showed that at a temperature of $10^{\circ} \mathrm{C}$ the growth of lilac microshoots practically stopped. For all that the reaction to low positive temperature turned out to be cultivar-specific (Churikova, Krinitsina, 2018). In nature, lilac buds pass by autumn to endodormancy. The decrease in temperature leads to this state when during further transfer to normal conditions active growth begins.

The understanding of peculiarities of lilac apexes' state in slow-growth culture is possible by comparison of the levels of expression of their genes in slow-growth conditions with those in conditions of natural (in vivo) dormancy and conditions of dormancy release. For this, it was necessary to assemble de novo the reference transriptome of Syringa vulgaris and to reveal possible differences in expression profiles in explants in vivo and in vitro.

\section{Materials and methods}

Two lilac cultivars were used for the experiment: 'P.P. Konchalovsky' and 'Velikaya Pobeda'. Both cultivars belong to D IV-III group (double, bluish), but 'P.P. Konchalovsky' has the corolla consisting of four petal circles, and 'Velikaya Pobeda', of two. Extraction of RNA for the reference transcriptome was carried out from 'P.P. Konchalovsky' apical complexes of vegetative shoots (apex with 2 leaf primordia) of this year when their active growth begins, and from the flowers at the stage of uncolored corolla. Samples (not less than 30 apexes for one sample) were fixed in RNA-later (Sigma). Also, data on the RNA sequence from apical complexes of vegetative buds during physiological dormancy were used.

For comparison of lilac's apexes in slow-growth culture (in vitro), during physiological dormancy (in vivo) and during active growth of plants on its end one-noded grafts of both cultivars were placed into slow-growth storage conditions $\left(+10^{\circ} \mathrm{C}\right)$ on MS medium with $1.5 \mathrm{mg} / 1$ 2-ip and $20 \mathrm{~g} / 1$ sucrose for 270 days.

For transcriptome analysis, apexes of one half of shoots were cut off and fixed in RNA-later. The remaining shoots were transferred to normal condition and allowed to grow for 28 days, after that active growth microshoot apexes were cut off and fixed in RNA-later, too. Likewise, the vegetative apices of adult $S$. vulgaris shrubs were collected under dormancy (in winter) and from active growth (in springtime).

The total RNA was purified with an RNeasy Plant Mini Kit (Qiagen) from $20 \mathrm{mg}$ of each fixed sample. The libraries 
were prepared using a NEBNext ${ }^{\circledR}$ Poly(A) mRNA Magnetic Isolation Module and a NEBNext ${ }^{\circledR U}$ Ultra RNA Library Prep Kit for Illumina (NEB) and sequenced using a HiSeq 2500 System. The reference transcriptome was assembled by Trinity (Haas et al., 2013), transcript quantification was made by Salmon. For annotation, transcripts were searched against the NCBI non-redundant (NR) protein database using BlastX, with a cut-off E-value of $10^{-5}$. Gene ontology (GO) terms were extracted from the annotation of high-score BLAST matches in the NCBI NR proteins database (E-value $\leq 1.0 \times 10^{-5}$ ) using Blast2GO (Conesa et al., 2005). Differential expression analyses were conducted using edgeR, a correction via false discovery rate (FDR) estimation was made.

\section{Results and discussion}

Using the Illumina RNA-seq technique, 263 million raw reads were obtained and $46.3 \%$ of them remained after adapter and quality filtering. The final de novo assemblies consisted of 281,159 transcripts. The length distribution of transcripts was as follows: 19 transcripts were longer than 1000 b.p. (3 of them are unique), 84,556 transcripts were in the range of 1000-9999 b.p. (10,429 of them are unique), 196,584 transcripts, in the range of 100-999 b.p. (28,308 of them are unique). 182,177 transcripts had blast hits, for 112,741 of them GO annotations were obtained.

Transcripts were categorized into 38 functional groups based on gene ontology categorization. Some of them belong to more than one group. Within this group, assignments to the molecular function category was highest with $80 \%$, followed by the biological process category $(69.5 \%)$ and the cellular component category $(58.3 \%)$. Under the molecular function category, binding $(59.78 \%)$ and catalytic activity $(64.77 \%)$ were the most common. A moderate number of transcripts $(10 \%)$ belong to the transporter activity group. A very small number of transcripts were associated with metallochaperone $(0.03 \%)$ and antioxidant $(0.02 \%)$ activity.

Among the biological process categories, metabolic process $(75.14 \%)$ and cellular process $(72.07 \%)$ were prominently represented. A moderate number of transcripts were also involved in the biological regulation (19.07\%), localization $(17.39 \%)$, regulation of biological process $(16.66 \%)$, response to stimulus (13.01\%), cellular component organization $(12.79 \%)$, cellular component biogenesis $(2.5 \%)$ and rhythmic process $(2 \%)$ categories, while only a limited number were associated with multi-organism process $(0.9 \%)$, development $(0.2 \%)$ biological adhesion $(0.1 \%)$, cell killing $(0.1 \%)$, growth $(0.08 \%)$, reproduction $(0.05 \%)$, locomotion $(0.05 \%)$, signaling $(0.05 \%)$ and reproductive process $(0.05 \%)$.

For the cellular component category, the majority of the transcripts were assigned to the cell $(60.09 \%)$, membrane $(58.52 \%)$ and organelle $(45.31 \%)$ categories. A moderate number of transcripts $(19.78 \%$ and $10 \%)$ belong to the protein-containing and macromolecular complexes.

A pairwise comparison of samples showed differences in the level of transcription of two $\beta$-glucosidase isoforms, five superoxide dismutase isoforms, four peroxidase isoforms and three xyloglucan xylo-glucosyltransferase isoforms in slowgrowth condition in vitro vs. endodormant lilac's plant apices in vivo. Differences in the level of transcription were identified for seven superoxide dismutase isoforms, three $\beta$-glucosidase isoforms and cytochrome p450 isoforms in lilac's shoot apices of active growth plants in vivo vs. in vitro.

$\beta$-glucosidase activity is connected with the response to abiotic stress through accumulation of antioxidant flavonols (Singh et al., 2015; Baba et al., 2017). Change in superoxide dismutase and peroxidase gene expression takes place during oxidative stress (Raychaudhur, Deng, 2000; Li et al., 2004). However, there were no differences in the expression of glutathione-S-transferases, other limitation oxidative stress proteins, which play a role in plant growth in vivo and shoot morphogenesis in vitro in Arabidopsis thaliana (Gong et al., 2005). Glutathione peroxidase was also absent among differentially expressed genes. They are considered to be important in plant growth and oxidative stress response (Bela et al., 2015). We think that differentially expressed thioredoxin in our case can compensate function of plant glutathione peroxidases due to similar molecular activities (Herbette et al., 2002).

\section{Conclusions}

Thus, a decrease in the temperature of lilac microshoot cultivation in sterile conditions down to $+10{ }^{\circ} \mathrm{C}$ leads to the same response as the response to oxidative stress. It keeps during active growth of shoots in sterile culture after transfer to standard conditions of cultivation $\left(+22^{\circ} \mathrm{C}\right)$.

\section{References}

Baba S.A., Vishwakarma R.A., Ashraf N. Functional characterization of CsBGlu12, a $\beta$-glucosidase from Crocus sativus provides insights into its role in abiotic stress through accumulation of antioxidant flavonols. J Biological Chemistry. 2017;292:4700-4713. DOI 10.1074/jbc.M116.762161.

Bela K., Horváth E., Gallé Á., Szabados L., Tari I., Csiszár J. Plant glutathione peroxidases: emerging role of the antioxidant enzymes in plant development and stress responses. J Plant Physiol. 2015;176:192-201. DOI 10.1016/j.jplph.2014.12.014.

Conesa A., Götz S., Garcia-Gomez J.M., Terol J., Talon M., Robles M. Blast2GO: a universal tool for annotation, visualization and analysis in functional genomics research. Bioinformatics. 2005;21:36743676. DOI 10.1093/bioinformatics/bti610.

Gabryszewska E. Effect of various levels of sucrose, nitrogen salts and temperature on the growth and development of Syringa vulgaris L. shoots in vitro. J Fruit Ornamental Plant Research. 2011; 19(2):133-148.

Gong H., Jiao Y., Hu W.W., Pua E.C. Expression of glutathione-Stransferase and its role in plant growth and development in vivo and shoot morphogenesis in vitro. Plant Mol Biol. 2005;57(1):53-66. DOI 10.1007/s11103-004-4516-1

Haas B.J., Papanicolaou A., Yassour M., Grabherr M., Blood P.D., Bowden J., Couger M.B., Eccles D., Li B., Lieber M., Macmanes M.D., Ott M., Orvis J., Pochet N., Strozzi F., Weeks N., Westerman R., William T., Dewey C.N., Henschel R., Leduc R.D., Friedman N., Regev A. De novo transcript sequence reconstruction from RNA-seq using the Trinity platform for reference generation and analysis. Nat Protoc. 2013;8(8):1494-512. DOI 10.1038/nprot.2013.084.

Herbette S., Lenne C., Leblanc N., Julien J.L., Drevet J.R., RoeckelDrevet P. Two GPX-like proteins from Lycopersicon esculentum and Helianthus annuus are antioxidant enzymes with phospholipid hydroperoxide glutathione peroxidase and thioredoxin peroxidase 
activities. European J Biochemistry. 2002;269(9):2414-2420. DOI 10.1046/j.1432-1033.2002.02905.x.

Li X.Q., Li S.H., Chen D.F., Meng F.R. Induced activity of superoxide dismutase and peroxidase of in vitro plants by low concentrations of ethanol. Plant Cell, Tissue Organ Culture. 2004;79:83-86. DOI 10.1023/B:TICU.0000049439.36866.4e.

Molkanova O.I., Churikova O.A., Konovalova L.N., Okuneva I.B. Clonal micropropagation of Syringa vulgaris L. introduced species. Vestnik Moskovskogo universiteta (Ser. Biol.). 2002;4:8-14. (in Russian)

Ozudogru E.A., Previati A., Lambardi M. In vitro conservation and cryopreservation of ornamental plants. In: Jain S., Ochatt S. (Eds.). Protocols for in vitro propagation of ornamental plants. Methods Mol Biol (Methods and Protocols). 2010;589. DOI 10.1007/978-160327-114-1_28.
Raychaudhur S.S., Deng X.W. The role of superoxide dismutase in combating oxidative stress in higher plants. Bot Review. 2000; 66(1):89-98 DOI 10.1007/BF02857783.

Refouvelet E., Le Nours S., Tallon C., Daguin F. A new method for in vitro propagation of lilac (Syringa vulgaris L.): Regrowth and storage conditions for axillary bud encapsulation in alginate beads, development of a preacclimatisation stage. Sci. Hortic. 1998;74:233241. DOI 10.1016/S0304-4238(98)00088-0.

Acknowledgements. The work is supported by the Scientific program AAAA-A16-116021660105-3 of the Department of Higher Plants, Lomonosov Moscow State University and by the Russian Academic Excellence Project 5-100 (Sechenov University).

Conflict of interest. The authors declare no conflict of interest. 